\title{
Automatic Car Parking System
}

\author{
Adnan M. Al-Smadi \\ Department of Electronics Engineering \\ Hijjawi Faculty for Engineering \\ Technology \\ Yarmouk University \\ Irbid, Jordan \\ smadi98@yu.edu.jo
}

\author{
Mofeed Msallam \\ Department of Electronics Engineering \\ Hijjawi Faculty for Engineering \\ Technology \\ Yarmouk University \\ Irbid, Jordan \\ 2014972039@ses.yu.edu.jo
}

\begin{abstract}
As the number of cars are increasing on roads, it becomes difficult to find a parking space. People drive around for half an hour or more to avoid facing this challenge. Automatic car parking is an autonomous car maneuvering system that takes the car from a traffic lane to a parking space. To provide more simplicity into people's lives, this paper will develop a system that enables car to park itself in parallel way. The system uses ultrasound sensors and Arduino microcontroller to detect the parking place automatically, and then moves the car to park in the accurate position. The system uses a four-wheel drive miniature vehicle to simulate the mechanism of a real car. This system becomes necessary for an inexperienced driver, especially in a narrow parking space without damaging his/her car or other cars nearby. In addition, disabled drivers can benefit from this technology. A prototype parking system that can perform parallel parking maneuvers autonomously has been implemented and tested. It performs as expected.
\end{abstract}

\section{Keywords-automatic parking, Arduino Microcontroller, ultrasonic sensors, H-bridge, parallel parking}

\section{INTRODUCTION}

With rapidly increasing number of cars, the problem of insufficient parking spaces is getting more complicated. In most cases, people drive around for half an hour or more to find a parking spot for their vehicles. In addition, the number of accidents caused by these cars has increased as a result of misalignment, especially among people who are new to roads. Reverse and trying to adjust a vehicle during parking is a complicated process especially in a crowded area and narrow parking space. Sometimes, frustrated driver who is unable to find a parking slot for his car a long a period of time may cause an accident. Automatic vehicle parking can complete parking processes safely and quickly without human intervention. In addition to reducing the probability of accidents caused by parking and saving lives. Hence, the purpose of automated car parking systems is to minimize human involvement as much as possible.

Car automation is fundamentally changing the concept of automotive manufacturing and industry. Nowadays, expensive cars such as Mercedes, Telsa, and BMW are built with self-parking feature [1]. Singh et al. [1], proposed a self-parking vehicle using semantic segmentation based on convolutional neural network. Razinkova et. al. [2] proposed an intelligent vehicle parking system that generates trajectory for parking using a fuzzy logic algorithm. Avgan [3], proposed an embedded fuzzy logic control algorithm for car self-parking. Azshwanth et al. [4] proposed a smart car parking model to help drivers in booking their parking slots beforehand and the car will be able to park automatically once it reaches the parking area. Liu et. al. [5] presented a research paper that develops a curricula and hands on laboratory to prepare for the future workforce needed for vehicle automation in automobile manufacturing and industry. Their paper extended to develop teaching materials for students projects on the subject of car automation. Ma et al. [6] proposed an automatic parking system based on parking scene recognition. They used machine vision and pattern recognition techniques to recognize parking scenario.

In this paper, we propose a method that enables a car to park itself in parallel way. The system uses ultrasound sensors and Arduino microcontroller to detect the parking place automatically. It controls the car movement and parking operations. The system moves the car to park in the accurate position. The system uses a four-wheel drive miniature vehicle to simulate the mechanism of a real car. Parking procedures and the corresponding algorithms are inspected and programed through Arduino microcontroller.

\section{PROPOSED SYSTEM}

As technology is growing fast, a lot of things are going automatic. The number of vehicles on the road is increasing on daily bases. This increase is causing traffic problems due to the fact that the available transportation infrastructure and parking facilities are unable to cope with the number of cars on the streets. To solve these problems, several solutions were proposed [1-6]. Automatic vehicle parking system is one solution.

This paper proposes an automatic car parking system using ultrasound sensors and Arduino 
microcontroller to detect the parking place automatically. The proposed system enables car to park itself in parallel way. Parallel parking is a method of parking a car in line with other parked cars or vehicles parallel to the road. Normally, parallel parking involves driving slightly passing the parking spot in parallel to the parked car in front of that spot. Then, turning the steering wheel while backing the car to park in the desired parking spot. This action may require several adjustments of forward and reverse moves. It requires relatively precise work and observations of the vehicle surroundings. Figure 1 shows a sample of parallel parking cars.

The main components of the proposed system in this paper consists of four Ultrasonic sensors, Arduino microcontroller, DC Motors, H-Bridge motor driver. These components are described as follows. The Block Diagram of the proposed system is shown in Figure 2.

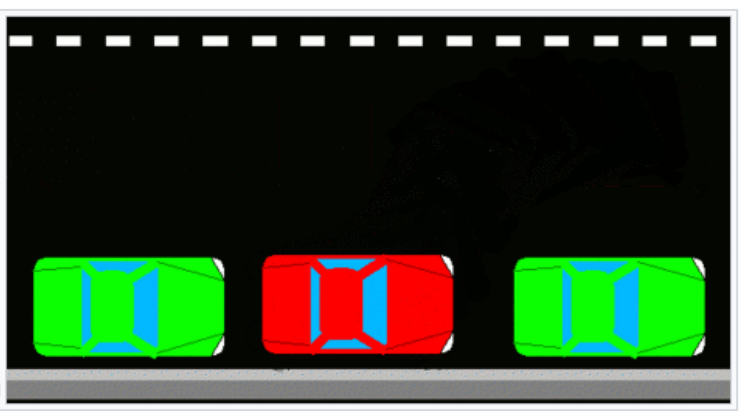

Fig. 1. A sample of parallel parking cars [7].

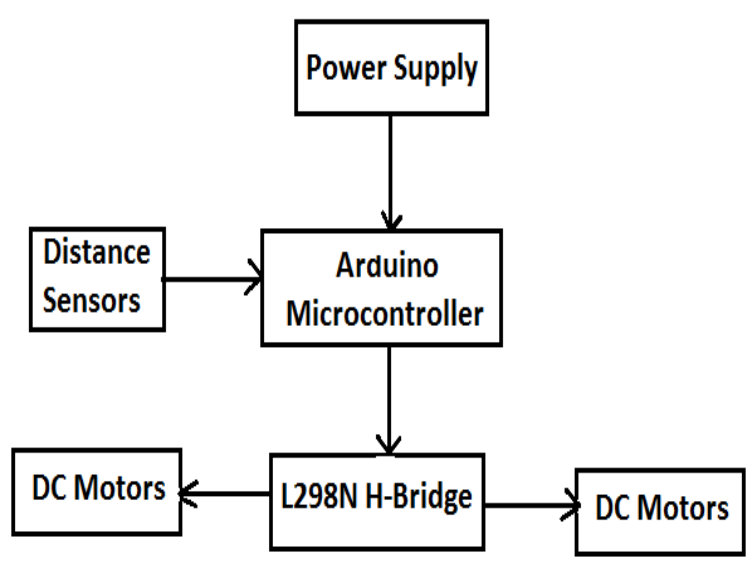

Fig. 2. Block Diagram for the proposed automatic car parking system.

\section{A. Arduino Microcontroller}

Arduino microcontroller is an open source board used to design and build electronic circuits. It is a lowcost device and is easy-to-use hardware and software [8].
Arduino boards are equipped with sets of digital and analog inputs/outputs pins. These boards are able to read inputs from sensors and other devices and turn them into an output which can activate other devices such as motors and LEDs. The Arduino microcontroller is shown in Figure 3.

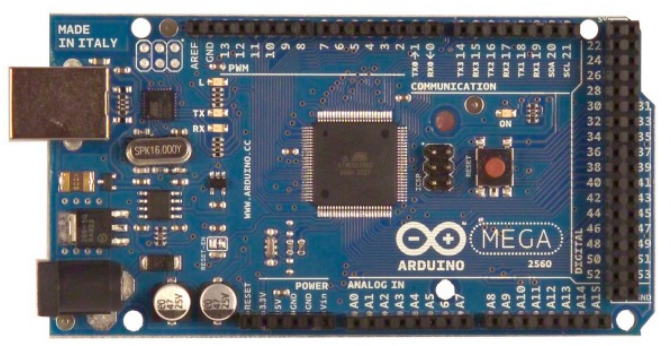

Fig. 3. Arduino microcontroller board [9]

\section{B. Ultrasonic Sensor}

Ultrasonic sensor is a transducer that works as sound transmitter and receiver. It uses ultrasonic waves to measure distance. It emits ultrasonic wave which then travels in the air. If this wave is detected by an object, it gets reflected back toward the ultrasonic sensor. It will be observed by the receiver [10]. The distance of a particular object can be calculated by measuring the time between sending a pulse and receiving an echo from boundaries of the given object.

$$
\text { Distance }=\mathrm{C} \times \mathrm{T}
$$

where $\mathrm{C}$ is the sonic speed and $\mathrm{T}$ is the time it takes from emission to reception. The time, $\mathrm{T}$, is divided by 2 because it is the time for go-and-return distance.

It is known that the universal speed of US wave at room conditions is $330 \mathrm{~m} / \mathrm{s}$. The time is measured using the inbuilt circuitry module. The distance is then calculated using the Arduino microcontroller. Figure 4 shows the function of the ultrasonic sensor.

The used HC-SR04 ultrasonic module has 4 pins, Ground, VCC, Trig and Echo. The ground pin should be connected to ground, while the VCC pin should be connected to the 5 volts pins on the Arduino board. The Trig and Echo pins are connected to any Digital I/O pin on the Arduino board. The connection of the ultrasonic and the Arduino board is shown in Figure 5.

In order to generate the ultrasound, the pin Trig should be set on High State for 10 microseconds which will send out an 8 cycle sonic burst. This will travel at the speed of sound and will be received back at the Echo pin. 


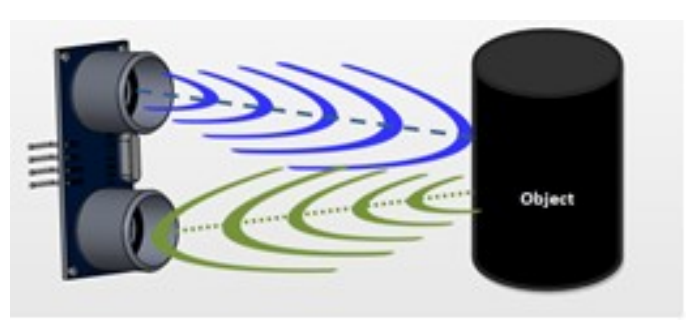

Fig. 4. Function of the Ultrasonic Sensor.

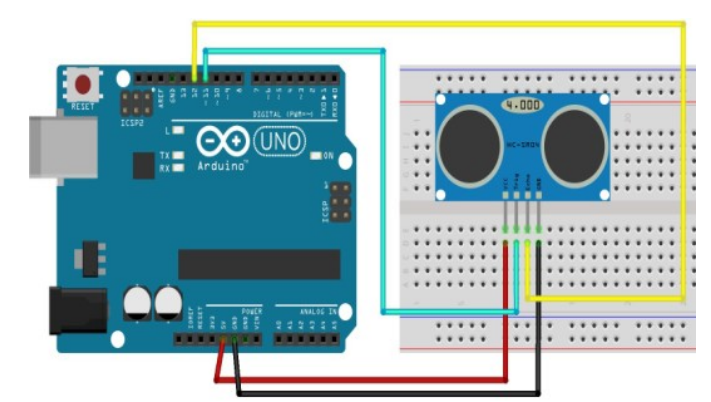

Fig. 5. The connection of the ultrasonic sensors and the Arduino board.

\section{H-Bridge}

An H-bridge is an electronic circuit that is used for controlling DC motor rotation. The H-bridge is designed to drive the motor clockwise and counter clockwise by switching the applied voltage. It switches the polarity of a voltage applied to a load to control a DC motor to run forward or backward. A basic Hbridge consists of four switches that allow controlling the applied voltage across a load in both directions. These switches are normally two npn and two pnp transistors [11].

Figure 6 shows the H-Bridge circuit with four switches. By activating two particular switches at the same time, the direction of the current flow can be changed. For example, when $S_{1}$ and $S_{4}$ are closed $\left(S_{2}\right.$ and $S_{3}$ are open), a positive voltage will be applied across the motor. On the other hand, when $\mathrm{S}_{1}$ and $\mathrm{S}_{4}$ are open switches (closing $S_{2}$ and $S_{3}$ ), the applied voltage is reversed, allowing reverse rotation of the motor.

The L298N is a dual H-bridge motor driver which allows speed and direction control of two DC motors at the same time. Figure 7 shows the L298N H-bridge. The connections for the H-bridge with Arduino and Motors are shown in Figure 8.

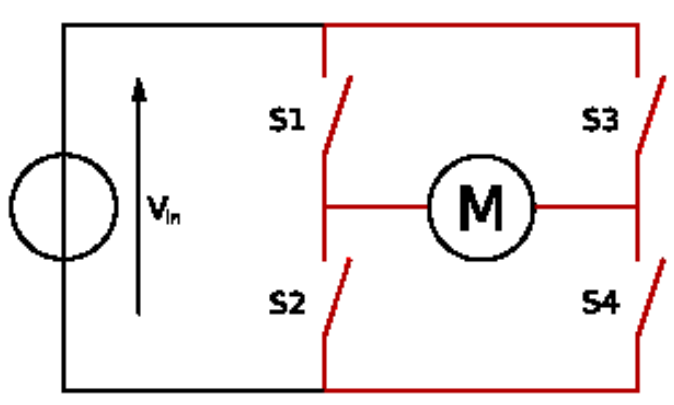

Fig. 6. H-Bridge Circuit.

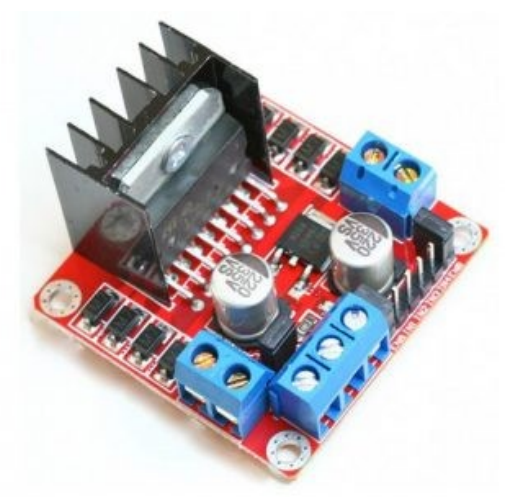

Fig. 7. L298N H-bridge [5].

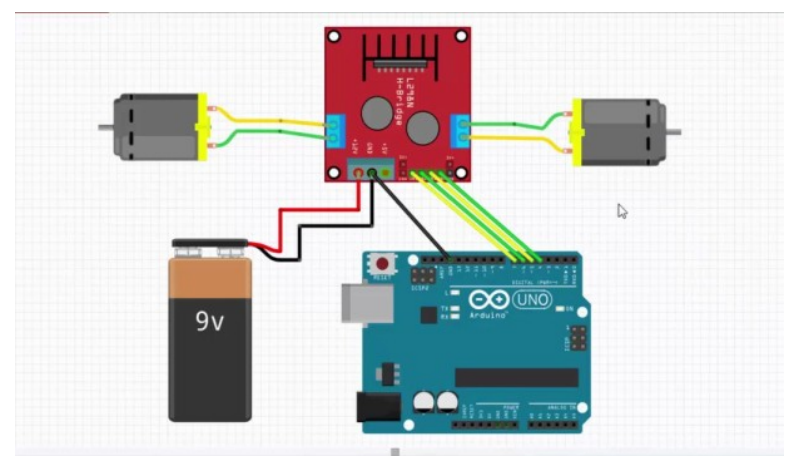

Fig. 8. H-bridge with Arduino and Motors

\section{RESULTS}

The automatic car parking system is controlled by the Arduino microcontroller. Figure 5 shows the connections of ultrasonic sensors with the Arduino board, while Figure 8 shows the connections of Arduino board with the L298N H-bridge and the DC motors. The ultrasonic sensors will detect if there is an obstacle and measure the distance between the car and the obstacle if it exists. It will inform the Arduino mocrocontroller. The system proceeds as follows. 
The particular car that looks for a parking space moves on the road along a straight line while measuring the distance on the left side of the car. If the distance is over a set threshold value, the car system assumes that it located a potential parking space. The car system starts measuring the distance it travels until the distance on its left side is less than or equal to the set threshold value. If the located spaces are large enough, then the parking sequence begins. A prototype parking system is shown in Figure 10.

The prototype starts by pressing the power switch to activate the automatic car parking system. The code in the Arduino microcontroller will run. The flow chart for the prototype parking system proceeds as shown in Figure 9. Hence, the four motors will receive the signal from the H-bridge to rotate the car forward. The ultrasonic sensor will detect if there is an obstacle and solid particles in front of the car. The Arduino microcontroller will calculate the distance continuously using Equation (1) until the distance equal or less than $7 \mathrm{~cm}$. Then, the car will stop, otherwise, the car will keep moving.

The parking mode depends on the four ultrasonic sensors. Firstly, the two-left sensors will search for a suitable place in order to park under the condition that there is no obstacle at the front side of the car.

The parking mode will be set when the measured distances from the two-left sensors are suitable for the length and width of the parking car. The length and the width of the prototype car are $(25 \mathrm{~cm}),(10 \mathrm{~cm})$, respectively.

If the parking mode is correct, the car will stop and rotate to the right 45 degrees. Then the car will move backward until the measured distance from the back sensor equals or less than $9 \mathrm{~cm}$. The car will rotate to the left 45 degrees move forward until the measured distance from the front sensor equals or less than $5 \mathrm{~cm}$. At this point, the car will stop as it parked in the desired slot. Table 1 shows the direction of the motors and the car. In Table I, CW represents clockwise, while $\mathrm{CCW}$ represents counter clockwise

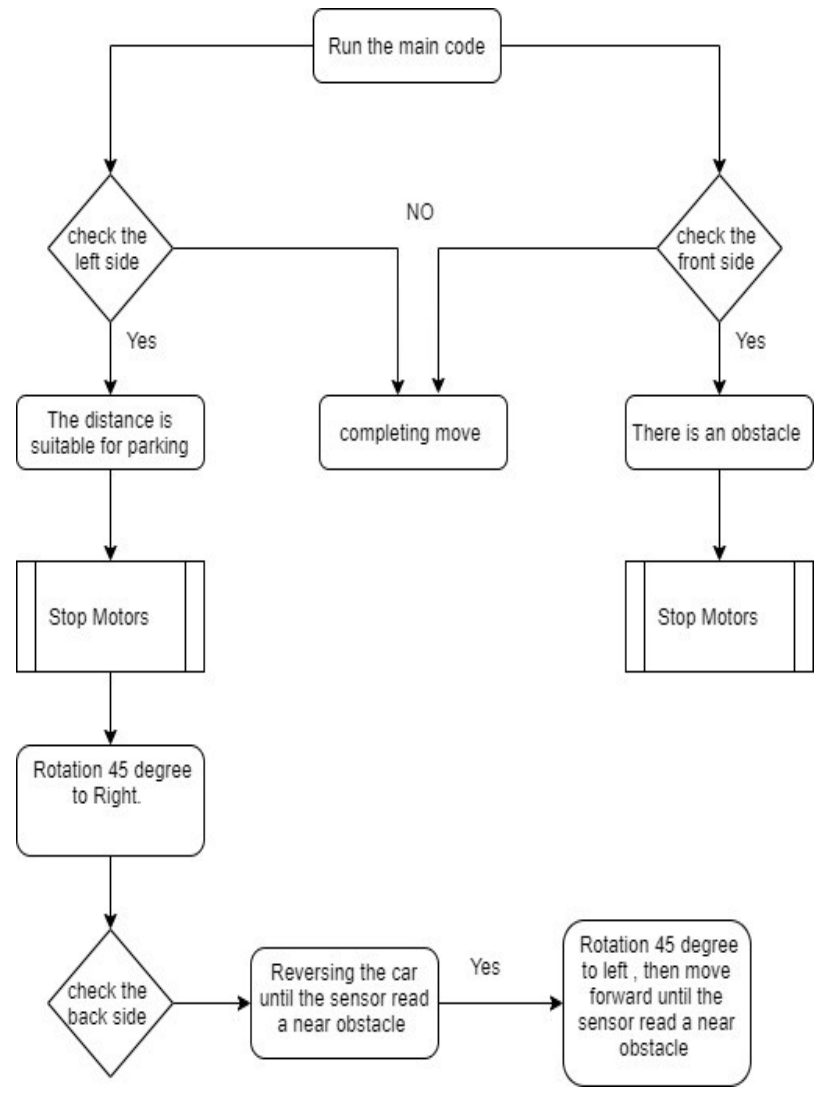

Fig. 9. A flow chart for the proposed automatic car parking system.

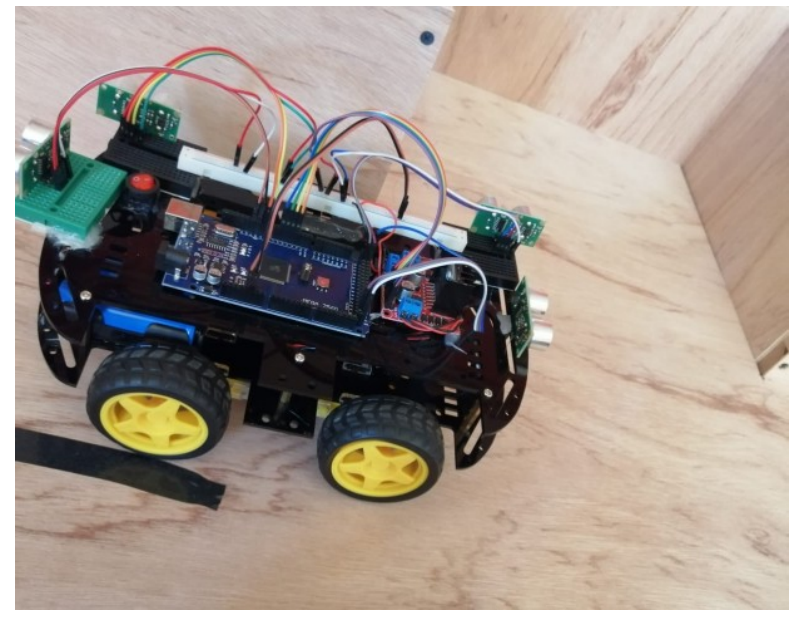

Fig. 10. A prototype parking system. 
TABLE I. THE DIRECTION OF THE MOTORS AND CAR.

\begin{tabular}{|c|c|c|}
\hline $\begin{array}{c}\text { Motor 1 } \\
\text { ( right motors) }\end{array}$ & $\begin{array}{c}\text { Motor 2 } \\
\text { (left motors) }\end{array}$ & Car \\
\hline CW & CW & Forward \\
\hline CCW & CCW & Backward \\
\hline CCW & CW & Right \\
\hline CW & CCW & Left \\
\hline Stop & Stop & Stop \\
\hline
\end{tabular}

\section{CONCLUSION}

In this pare, a model for an automatic car parking system was proposed. The system uses ultrasound sensors and Arduino microcontroller to detect the parking place automatically, then moves the car to park in the accurate position. The system uses a fourwheel drive miniature vehicle to simulate the mechanism of a real car. A prototype parking system that can perform parallel parking maneuvers autonomously has been implemented and tested. It performs as expected.

\section{REFERENCES}

[1] Anukriti Singh, Aditi Chandra, Divya Priyadarshni, and Nisheeth Joshi, "Self Parking car prototype," International Conference on Advances in Computing, Communications and Informatics (ICACCI), 19-22 September, Bangalore, India, pp. 2251-2254, 2018.

[2] Anastasia Razinkova1, Hyun-Chan Cho1, and Hong-Tae Jeon, "An Intelligent Auto Parking System for Vehicle," International Journal of Fuzzy Logic and Intelligent Systems, vol. 12, no. 3, pp. 226-231, 2012.

[3] Utku Avgan, "Design and Control of self-Parking Model Car," Master Thesis, Department of Mechanical Engineering, Middle East Technical University, Turkey, 2003.

[4] D.Azshwanth, Mithul Titten Koshy, and Mr.T.Balachander, "Automated car parking system." International Conference on Physics and Photonics Processes in Nano Sciences, 20-22 June, Eluru, India, Conference Series Volume 1362, pp. 1-9, 2019.

[5] Honghong Liu, Gene Yeau-Jian Liao, Chih-Ping Yeh, and Jimmy Ching-Ming Chen, "Automatic Parking Vehicle System," ASEE's 123rd Annual Conference and Exposition, 26-29 June, New Orleans, LA, pp. 1-12, 2016.

[6] Shidian Ma, Haobin Jiang, Mu Han, Ju Xie, and Chenxu Li, "Research on Automatic Parking Systems Based on Parking Scene Recognition," IEEE Access, Vol. 5, pp. 21901- 21917, 2017.

[7] https://en.wikipedia.org/wiki/Parallel_parking.

[8] S. Monk, Programming Arduino, McGraw-Hill Companies, New York, 2012.

[9] Adnan Al-Smadi, Wasan Al-Ksasbeh, Mohammad Ababneh, and Manar Al-Nsairat, " Intelligent Automobile Collision Avoidance and Safety System"17th IEEE International MultiConference on Systems, Signals \& Devices (SSD20), Sfax, Tunisia,(in webinars), July 20-23, 2020.
[10] Alessio Carullo and Marco Parvis, "An ultrasonic sensor for distance measurement in automotive applications," IEEE Sensors Journal, vol. 1, No. 2, pp. 143 - 147, 2001.

[11] T. Ozer, S. Kivrak, and Y. Oguz, "H Bridge DC Motor Drive Design and Implementation with using dsPIC30f4011," International Journal of Innovative Research in Science, Engineering and Technology, Vol. 6, No. 10, pp. 75-83, 2017.

\section{Creative Commons Attribution License 4.0 (Attribution 4.0 International, CC BY 4.0)}

This article is published under the terms of the Creative Commons Attribution License 4.0 https://creativecommons.org/licenses/by/4.0/deed.en_US 